\title{
Towards an Irritable Bowel Syndrome Control System Based on Artificial Neural Networks
}

\author{
Ina Podolski and Achim Rettberg \\ Carl von Ossietzky University Oldenburg, Germany, Eschwerg 2 \\ 26121 Oldenburg, Germany \\ \{Ina.Podolski, Achim.Rettberg\} @iess.org
}

\begin{abstract}
To solve health problems with medical applications that use complex algorithms is a trend nowadays. It could also be a chance to help patients with critical problems caused from nerve irritations to overcome them and provide a better living situation. In this paper a system for monitoring and controlling the nerves from the intestine is described on a theoretical basis. The presented system could be applied to the irritable bowel syndrome. For control a neural network is used. The advantages for using a neural network for the control of irritable bowel syndrome are the adaptation and learning. These two aspects are important because the syndrome behavior varies from patient to patient and have also concerning the time a lot of variations with respect to each patient. The developed neural network is implemented and can be simulated. Therefore, it can be shown how the network monitor and control the nerves for individual input parameters.
\end{abstract}

Keywords: Medical Applications, Irritable Bowel Syndrome, Artificial Neural Networks.

\section{Introduction}

Medical support for example to remote supervision and monitoring by automated devices for control medication and treatment is a new trend in medical systems. The development of nano-devices offers the freedom to realize monitoring and controlling of specific functionalities. Especially for human's, nano-devices can play an important role to support their life in the case of diseases, pain or illness. Complex tasks of the human body can be supported with nano-devices. They can be implanted into the body to observe internal functionalities. Besides measurement such devices can control and therefore help the human body to overcome the critical situations. For example, if a system is able to measure enzymes, hormones, and temperatures and to observe the nerves of the large intestine it is possible to control the irritable bowel syndrome (IBS). We assume the availability to observe those parameters. Then we are able to set up such a system by implant small nano-devices. On every device an algorithm based on an artificial neural network (ANN) is executed. These networks are able to learn and adapt themselves. This is necessary for IBS, because these symptoms differ for every patient and are changing over time. 
The paper is structured as follows. In section 2 the IBS problem is briefly described. Afterwards, in section 3 the biological nerves system respectively the parts of the human nerves system involved in IBS is presented. This description is necessary to get a notion of the complexity of the nerves. Therefore, the different nerves and their correlation are shown. Section 4 discusses the control method based on an ANN. There the interaction with the human nerves and our system is given. The paper is closed by a summary and outlook given in section 5 . This paper gives an overview of the possibilities for IBS control from a computer scientist perspective. We had a lot of discussions with doctors and medical scientist working in this area and they are highly interested in this idea.

\section{Irritable Bowel Syndrome}

The irritable bowel syndrome (IBS) is a dysfunction of the digestive tract with chronic medical conditions. In the last decades IBS has been dramatically growing. Meanwhile a fair intestine is an exception. In Germany an approximation shows that more than five million people have IBS or large intestine problems [7]. Symptoms vary and change from time to time. Between the intestine and the human brain exist a strong connection. A network of nerves interconnects the nerve plexus of the digestive tract with the central nervous system in the head. Information between the state of the intestine and the brain will permanently exchange. In the case of a fair intestine the human is not recognizing this information flow. Humans with IBS have problems with this information exchange from intestine to brain, because the nerves are seriously overestimated. Furthermore, the nerves threshold is decreasing with the effect of a really painful digestion. The autonomic nervous system, as well as hormones and enzymes in the intestine play an important role of this disorder [1][6]. This will be discussed in detail in the next section.

\section{Human Nervous Systems}

In this section the structure of the central nervous system (CNS) and the peripheral nervous system (PNS) is described. We call our system Intestine-Nano-Attack (INA). The human body has two nerves systems: the central and peripheral nervous system. Figure 1 gives an overview of the nervous systems involved in the context of this paper. The communication and the tasks of the nerves will be described in detail in this section.

The peripheral nervous system operates mainly autonomous and independent from the CNS, some decisions are controlled by the CNS and other ones are locally handled. It consists out of a congregation from nerve cells and quiet more compared with the spinal cord.

The PNS is responsible for the organ smooth muscles, the heart and the glands. Smooth muscles are inside the organs and are without arbitrary control like the stomach and intestine. The PNS consists out of three components: the sympathetic nervous system (responsible for the activation), the parasympathetic nervous system (responsible for the passive states) and the enteric nervous system (ENS). The ENS is known 


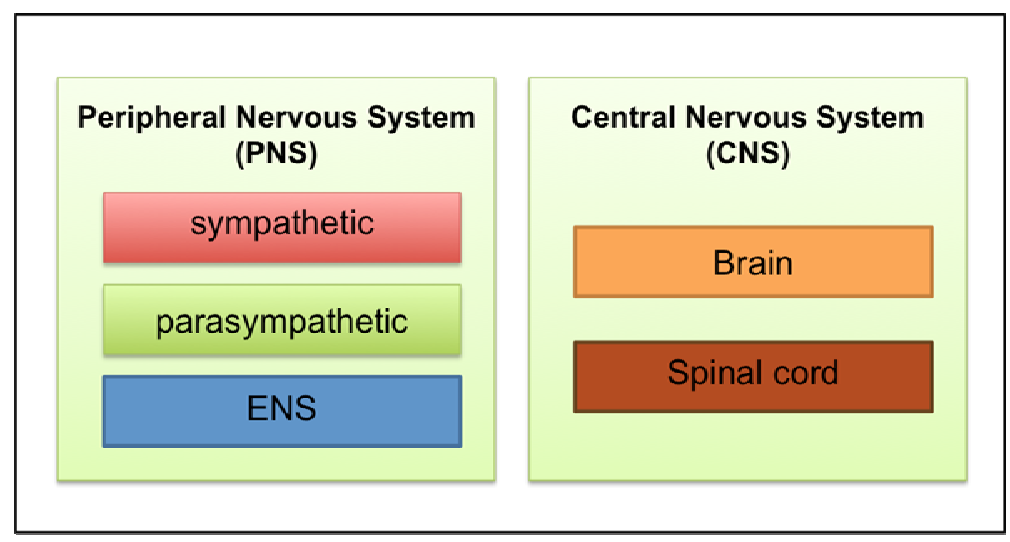

Fig. 1. Human nervous system.

as the second brain by researchers [3]. Therefore, to reduce IBS symptoms the monitoring and control of the PNS and all involved nervous systems are in the focus of this work.

There exist no direct connection between CNS and ENS. The ENS consists of vegetative nerve fiber in the wall of the intestine. The second brain consists on one hand out of a nerve fiber system between the muscles layers of the intestine and on the other hand of a nerve congregation underneath the intestine mucosa. The tasks of these nerves are: analysis of nutrition components, coordination of intestine motility, control of the glands, control of the liquid and food intake and finally the control of the immune system.

The second brain isn't only based on nerve impulse furthermore it produces intensively neuron transmitters. Every chemical messenger produced by the brain is also used inside the intestine and his second brain. There is about $97 \%$ of the serotonin a hormone of the human body is produced by the intestine. The PNS is really fast with the production of the chemical messengers. Control cycles need only milliseconds for the activation. In opposite to this hormones have a longer reaction cycle and responsible for the global change of the intestine [3].

Usually ENS is a stable system, but nevertheless due to different causes vegetative dysfunction can appear. There are no structural changes, but functional interferences. Right now we will focus only on nerves with our system, later on we will extend our approach by measuring hormones and enzymes. In the following we will discuss the nervous system and characteristics of the involved neurons.

The ENS and spinal cord consists of different neuron types like motor neurons, inter and sensory neurons. Table 1 shows the differences between the neurons respectively their applications and properties. Additionally the neurons can be divided by their location in the spinal cord. The motor neuron is the neuron that could be easily detected. The cell bodies of the motor neuron connected with the body muscles are located in the spinal cord. They hand over the neuronal information to the muscles and influence the activity of the human body. 
Table 1. Neuron types and their applications

\begin{tabular}{|c|c|c|}
\hline Types of neurons & Property & Application \\
\hline Inter neuron & Structure element & Structural filter \\
\hline Sensory neuron & Stimulus receiver \& filter & Signal absorption \\
\hline Motor neuron & Stimulus sender \& filter & Signal dispensary \\
\hline
\end{tabular}

An inter neuron is a nerve from the CNS, that connects two nerve cells. It is also known as switch neuron. The inter neuron is a sensory and activity neuron that modifies the information between nerve cells.

The dorsal horn is the part of the spinal cord that receives painful nerve impulse. The dorsal spinal cord handles different impulses like pain, temperature or mechanical pressure recognized by the sensory nerve cells. An important sensory neuron is the pain receptor, called nociceptor. They are specialized nerve endings located throughout the body in most body tissues. Once the nerve endings are stimulated they begin firing the nerves that are connected to them and send pain signals to the spinal cord and brain. [2].

Nociceptors of the intestine are activated by extension and contraction. If the impulse reaches a specific bound an action potential will be activated and directed by the nerves fibre to the spinal cord. Afterwards the information reaches the brain and the human recognizes the pain of the intestine. All levels of the CNS have pain blocking mechanism systems for modulation of the pain. In the case the CNS is confronted with steady pain signals, a lot of complex changes within the peripheral nerve system and CNS happens. Still inactive nociceptors are activated, new proteins are produced and tremendous changes on molecular biological level will take place. This often vividly changes in the CNS leads to a pain memory. The pain outlasts the damaging event for days, weeks, months or years. This leads to chronic pain that is typical for IBS patients.

Chronic pain occurs when the back part of the spinal cord has been bombarded by severe pain for a long time, see figure 2 . The inter neuron adjusts and transforms to the wind-up nerve by adding fast pain receptors that amplify the pain signal and pass the amplified signal to the nerve tract that runs up to the brain. From there, the thinking and emotional centers of the brain receive a large pain signal and the mind perceives increasingly severe intractable pain. Modifier pathways in the brain attempt to tone down the pain, but are defeated at the back part of the spinal cord by the wind-up nerve. The brain keeps receiving amplified pain signals and the mind continues to perceive unrelenting and severe pain [2].

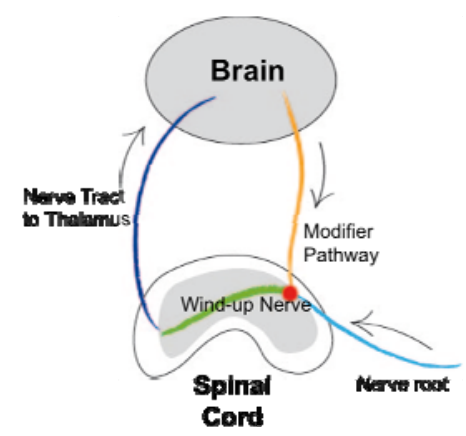

Fig. 2. Remaining pain 


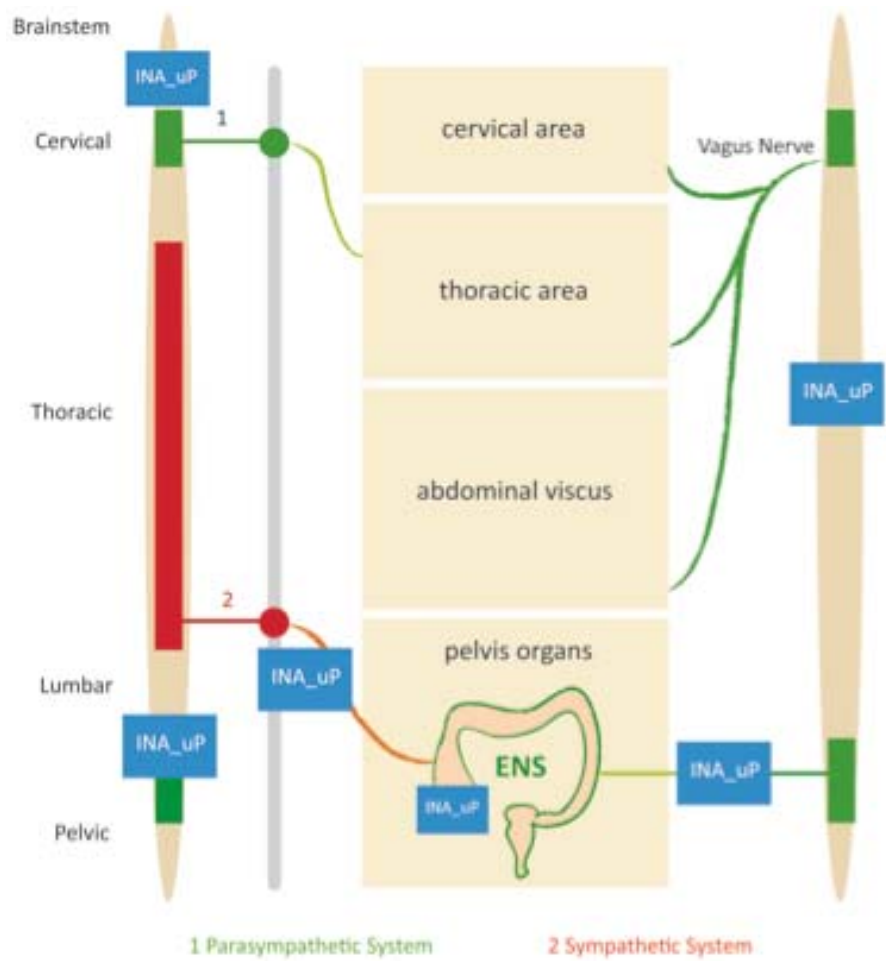

Fig. 3. Communication between nerves and organs and the monitoring and control with the INA system

Figure 3 shows the communication between nerves and organs. The composition of the nerve fibres to the organs will be explained in the following paragraph. Additionally we will show how the nerves influence the organs.

The sympathetic nervous system is richly connected to the thoracic and peripheral organs. Many levels of sympathetic nerves connect with cell bodies in the spinal cord. Pain can become coupled with the sympathetic nerves causing a dramatic central pain [2].

The cranial nerve called vagus nerve emerges directly from the brain stem in contrast to spinal nerves, which emerge from segments of the spinal cord. The vagus nerve serves the head and cervical with parasympathetic fibre and furthermore the thoracic organs as heart, stomach and digestive tract, but except the colon.

The system presented in this paper is able to measure, observe and stimulate parts of the sympathetic, parasympathetic and the ENS to reduce IBS based pain and convulsion. Several nano systems located inside the human body build the hardware part of the system (see figure 3). The software running on a nano-device or microprocessor for the INA system is based on ANN. This is described in the next section. 


\section{Control Method Based on Artificial Neural Nets}

The proposed control method used inside the INA system is based on neural nets [8], [9] and [10]. As already known artificial neural nets (ANN) are a biological inspired model consisting of a graph with neurons as nodes and edges as connection elements. There exist different types of ANNs. Here Kohonen nets [11] are a perfect match for the application, because this net allows unsupervised learning, which is really important for the INA system control.

In figure 4 we see inputs in form of patterns for the ANN. Additionally, neurons in the figure correspond to circles, hormones are ellipses, enzymes are triangles and finally proteins are diamonds. The net may learn similar patterns to identify them and combine them to groups. Afterwards, the trained net is able to find patterns and have to ensure correct selection/learning of new and similar patterns. The learning of similar patterns is important for our approach, because as we discussed previously IBS patterns vary. To ensure the correct execution the knowledge of the training phase is stored within the neurons. We test the net with similar pattern of the same class that are similar but not equal to the training patterns. In figure 4 for example we can see that neurons corresponds to circles, but the size of the circle vary. Our net has to learn that a circle may have different sizes, which means an element may has different characteristics.

IBS control requires self-organization, so to learn new pain patterns and to react on it without an observation for each evaluation step. For safety reasons such a system can end up in a chaotic state, but this can be avoided by the error detection.

The Kohonen net support exactly this behavior the INA system needs. Unsupervised learning implies self-organization and the Kohonen net starts initially with a socalled self-organizing map.

In our case this map is constructed from typical pattern by measuring the nerves, hormones and enzymes. With a cluster analysis [10], [11] the net is pre-partitioned into clusters (see figure 5). The cluster will show regions consisting of different types of neurons. Regular neurons correspond to normal situation inside the human body

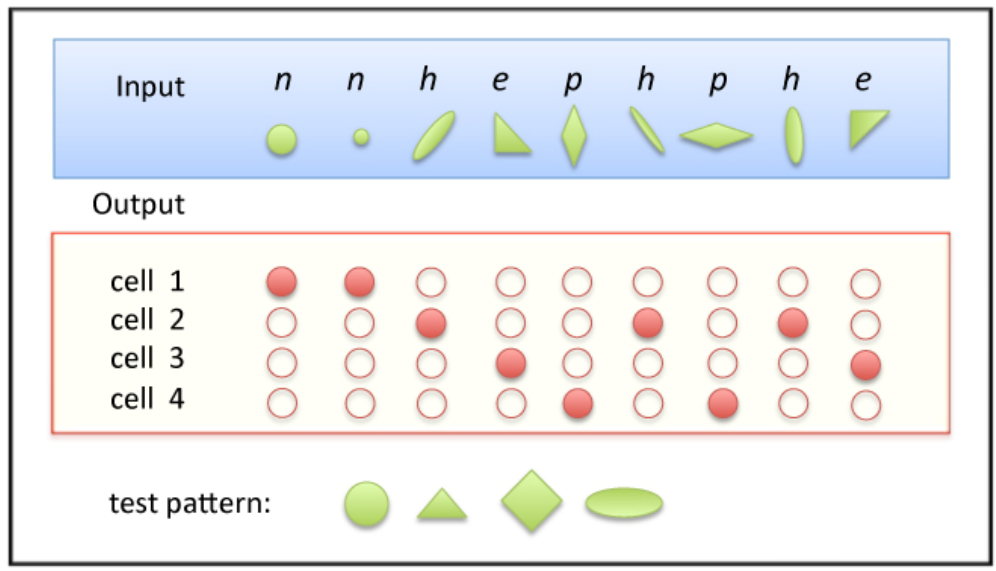

Fig. 4. Training pattern with neurons (n), hormones (h), enzymes (e) and proteins (p) 

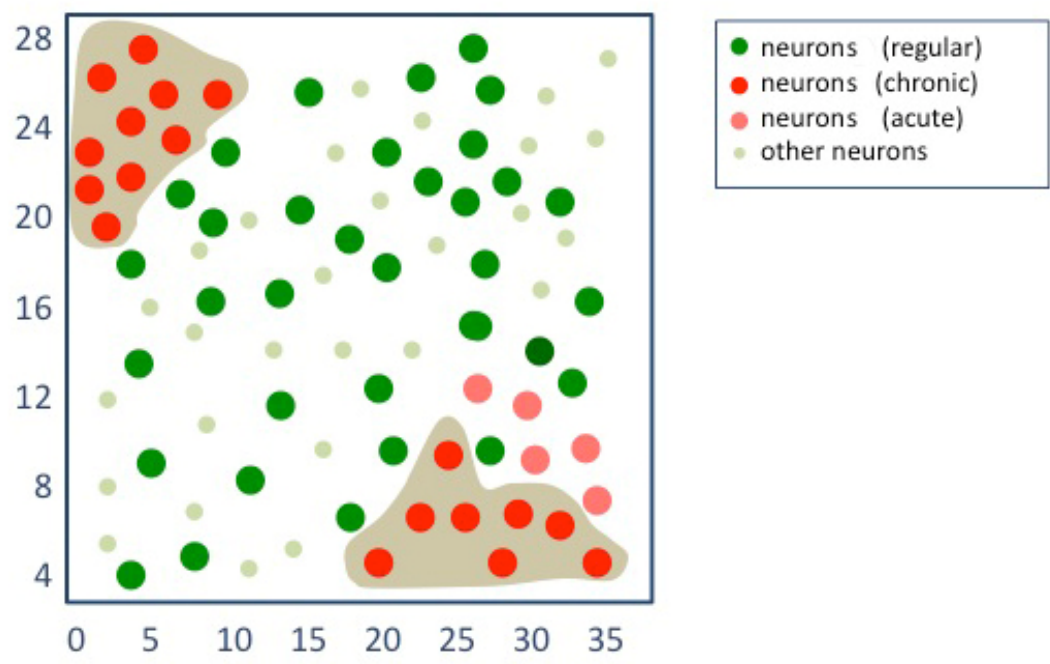

Fig. 5. Learning and finding patterns by recognizing cluster points

without IBS attacks. Acute pain from IBS are represented by acute or chronicle neurons. The difference between both is described in section 3. The other neurons in figure 5 are not related with IBS. The cluster analysis ensures that neurons with similar behavior built a region in the self-organizing map. For the initial clustering the weight of the neurons is calculated. The value of the weight inherits the measured nerves, enzymes and hormones.

Figure 6 shows an abstract implementation of the INA system. The clustered selforganizing map is the initial net of the neural network block. For each input pattern the system calculates the weight by the same calculation as for the neurons of the initial net. Afterwards the learning algorithm of the Kohonen net matches the input pattern by similarity to a neuron within the self-organizing map. Usually, the Euklidean distance is calculated for the input pattern and each neuron. The neuron with weight most similar to the input is called the best matching unit [11]. Afterwards, the weights of the best matching unit and neurons close to it in the self-organizing map are adjusted towards the input pattern. Afterwards, the weights of the best matching unit and neurons close to it in the self-organizing map are adjusted towards the input pattern, if the input is a good candidate to adapt the net. The decision if an input pattern is good or not is done by the error detection unit, see figure 6 . Therefore, the error detection tries to ensure an optimal adaptation of the self-organizing map. Within the error detection the similarity according the nerves, hormones and enzymes between the best matching unit and the input is compared. If the similarity is given the best matching unit and neurons close to it is adjusted otherwise not. The process described above is repeated for each input pattern. Obviously the INA system is able to adapt over time to normal and typical IBS patterns. 


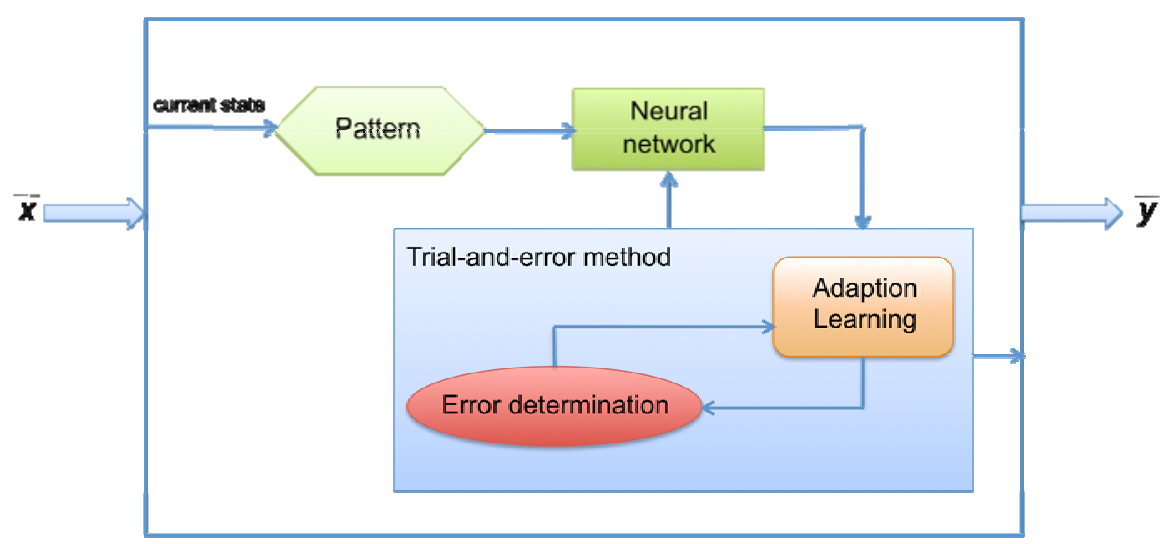

Fig. 6. The INA system

The ANN of the INA system consists of neurons that correspond to nerves of the nervous system. For every measured value and for all necessary outputs an input respectively output neuron is created. From the nerves (parasympathetic, sympathetic, vagus nerve), the temperature within the large intestine, the hormones and enzymes a feature vector (input) is built.

The error detection method is good to handle the complexity and correlation. The method helps to adjust an optimal adaptation of the net to the problem. The INA system finds optimal counteractions to avoid chronic pain of IBS patients. Counteractions may be a temperature adjustment in the large intestine, a hormones and enzymes control and nerves stimulation so that a typical IBS pattern is blocked. By blocking the IBS pattern the patient is not confronted with chronic pain from IBS. This was exactly the goal of the INA system.

\section{Conclusion and Future Work}

Within this paper we presented an embedded medical system to help patients with IBS. Several so-called nano-devices or microprocessors with INA system running could be used in the body to measure and influence the nerves involved in IBS. IBS is a complex problem and observing nerves it not sufficient enough, therefore, our system controls additionally the temperature, hormones and enzymes inside the large intestine. The control algorithm based on an ANN that offers the freedom of adaptation and learning of the control algorithm. By the artificial network our system is able to train especially for the detection of IBS typical patterns and not to touch other pain problems in the large intestine. Up to now, our approach is an idea and partly implemented. We contacted medical scientist and they are really interested. We set up the ANN and start with the training of the net and the simulation. Our approach is a realtime system that reacts efficiently by parallel execution on partial patterns. It is failure tolerant in the case of errors and besides this it can be interpreted as an early warning system.

Future work will be a complete software implementation of the ANN to make a deeply training of the network. Additionally, we plan to set up a hardware 
demonstrator with an evaluation board and several sensors to proof that the ANN can be implemented in an embedded system.

\section{References}

1. Novartis Pharma Schweiz, Irritable Bowel Syndrome (Reizdarm - Überblick) (2001)

2. http://www. bayareapainmedical.com/

3. http://www. charite.de/anatomie/lehre/skripte/vegetativ.htm

4. http://wwwmath. uni-muenster.de/SoftComputing/lehre/material/ wwwnnscript/prin.html

5. http://www. neuronalesnetz.de/units.html

6. http://www.krankerfuerkranke.de/archivgesundheit/ Rheuma_Reizdarm.htm

7. Weiß, T.: Fibromyalgie: Das erfolgreiche Ernährungsprogramm. Südwest-Verlag (2008)

8. Kratzer, K.P.: Neuronale Netze - Grundlagen und Anwendungen. Carl Hanser Verlag, München (1999)

9. Rey, G.D., Wender, K.F.: Neuronale Netze: Eine Einführung in die Grundlagen, Anwendungen und Datenauswertung. Huber Verlag, Bern (2008)

10. Yoshida, M., Sato, H.: New Research on Neuronal Networks, Nova Science Pub. Inc., Bombay (2008)

11. Kohonen, T.: Self-Organizing Maps. Springer, Berlin (1995) 